\title{
TWO EXAMPLES OF BOREL PARTIALLY ORDERED SETS WITH THE COUNTABLE CHAIN CONDITION
}

\author{
STEVO TODORCEVIC
}

(Communicated by Andreas R. Blass)

\begin{abstract}
We define an open symmetric two-place relation on the reals such that the reals cannot be covered by countably many sets of related elements, but there is no uncountable set of unrelated elements. The poset $\mathscr{P}$ of finite sets of related elements satisfies the countable chain condition but it may fail to have the property $\mathrm{K}$, i.e., a substantial irregularity can be injected in $\mathscr{P}$.
\end{abstract}

We construct two examples of Borel posets that satisfy the countable chain condition but violate certain natural strengthenings of this condition. The first example, discovered by the author several years ago to answer a question of $L$. Harrington and S. Shelah, is a Borel version of the poset presented in $[5,9.10]$. Let $\pi \mathbb{Q}$ be the set of all subsets of the rationals ordered by $x<y$ iff there is a $q$ in $y$ such that $x=\{p \in y: p<q\}$. Let $\mathscr{P}_{0}$ be the set of all finite antichains of the pseudotree $\pi \mathbb{Q}$. The ordering of $\mathscr{P}_{0}$ is the inclusion. Clearly, $\mathscr{P}_{0}$ is Borel.

Claim 1. $\mathscr{P}_{0}$ is a ccc poset.

Proof. Almost identical to the standard proof for the poset of all finite antichains of an Aronszajn tree. (See [5, 9.10(iii)].)

Claim 2. $\mathscr{P}_{0}$ is not $\sigma$-linked.

Proof. Assume $\left\{\mathscr{L}_{i}\right\}$ is a sequence of linked subsets of $\mathscr{P}_{0}$. Let $q_{0}$ be any rational, say $q_{0}=0$. If there is $\{t\}$ in $\mathscr{L}_{0}$ such that $\sup t<q_{0}$, pick one and call it $t_{0}$; otherwise $t_{0}=\{-1\}$. Pick a rational $q_{1}$ such that $\sup t_{0}<q_{1}<q_{0}$. If there is $\{t\}$ in $\mathscr{L}_{1}$ such that $t_{0} \lessdot t$ and $\sup t<q_{1}$, pick one and call it $t_{1}, \ldots$, and so on. Let $t_{\infty}=\bigcup_{i} t_{i}$. Then $\left\{t_{\infty}\right\} \notin \mathscr{L}_{i}$ for all $i$.

The second example is a Borel version of the Galvin-Hajnal poset as presented in [2]. A similar modification with a quite different motivation is given in [1]. The basic properties of the poset, however, are based on a completely different set of ideas $([3, \S 4],[4, \S 2])$. Let $\mathscr{S}$ be the set of all converging sequences of

Received by the editors December 11, 1989 and, in revised form, September 10, 1990.

1980 Mathematics Subject Classification (1985 Revision). Primary 03E05, 03E15; Secondary $54 \mathrm{H} 05$.

Research supported in part by NSF Grant DMS- 8505550 . 
the reals which do not contain their own limits and let $\mathscr{P}_{1}$ be the set of all finite subsets $p$ of $\mathscr{S}$ such that $\lim s \notin t$ for all $s$ and $t$ in $p . \mathscr{P}_{1}$ is ordered by the inclusion. It is clear that $\mathscr{P}_{1}$ is Borel.

Claim 3. $\mathscr{P}_{1}$ is $c c c$.

Proof. Suppose we have an $\omega_{1}$-sequence $\left\{p_{\alpha}\right\}$ of elements of $\mathscr{P}_{1}$. Let $F_{\alpha}=$ $\left\{\lim s: s \in p_{\alpha}\right\}$. We may assume $F_{\alpha}$ 's have some fixed size $n$ and that some fixed finite sequence $I_{0}, \ldots, I_{n-1}$ of rational intervals separates points of $F_{\alpha}$ 's from sequences of $p_{\alpha}$ 's not converging to them. We may also assume that $F_{\alpha}$ 's form a $\triangle$-system with root $F$. Note that in finding two compatible conditions, sequences converging to a member of $F$ do not cause any difficulty, so we may assume $F=\varnothing$. We may also assume that $\alpha<\beta$ implies that no $s$ of $p_{\alpha}$ contains a point from $F_{\beta}$. Thus, we are in the situation of a closed set-mapping on a set of reals which respects a well-ordering of that set ([3, Lemma 13], [4, $\S 7])$, but for the convenience of the reader we shall reproduce the argument. Identify $F_{\alpha}$ with the element of $\mathbb{R}^{n}$ which enumerates it according to the usual ordering of $\mathbb{R}$. Fix $\gamma<\omega_{1}$ such that $\left\{F_{\alpha}: \alpha<\gamma\right\}$ is dense in $\left\{F_{\alpha}: \alpha<\omega_{1}\right\}$ and choose $\delta>\gamma$. Then for $i<n, J_{i}=I_{i} \backslash \bigcup p_{\gamma}$ is an open set containing the $i$ th element of $F_{\delta}$. So there must be $\beta<\gamma$, such that for all $i<n$, the $i$ th element of $F_{\beta}$ is in $J_{i}$. Then $p_{\beta}$ and $p_{\gamma}$ are compatible.

Claim 4. $\mathscr{P}_{1}$ is not $\sigma$-linked.

Proof. Assume $\left\{\mathscr{L}_{i}\right\}$ is a sequence of linked subsets of $\mathscr{P}_{1}$. Pick a real $r_{0}$. If there is increasing $t$ with $\{t\}$ in $\mathscr{L}_{0}$ such that $\lim t<r_{0}$, pick one and call it $t_{0}$. Otherwise, $t_{0}$ is arbitrary increasing with $\lim t_{0}<r_{0}$. Pick $r_{1}<r_{0}$ above $\lim t_{0}$. If there is an increasing $t$ with $\{t\}$ in $\mathscr{L}_{1}$ such that $\lim t_{0} \in t$ and $\lim t<r_{1}$, pick one and call it $t_{1}, \ldots$, and so on. Let $t_{\infty}=\left\{\lim t_{i}\right\}$. Then $\left\{t_{\infty}\right\} \notin \mathscr{L}_{i}$ for all $i$.

Claim 5. If $\mathfrak{b}=\omega_{1}$, then $\mathscr{P}_{1}$ does not have property $K$.

Proof. In [4, 2.1], we have produced a set of reals $A$ of size $\aleph_{1}$ and for each $a$ in $A$, a sequence $H(a)$ converging to $a$ such that for every uncountable $B \subseteq A$ there exist $a \neq b$ in $B$ such that $a \in H(b)$. This means that $\{\{H(a)\}: a \in A\}$ as a subset of $\mathscr{P}_{1}$ does not contain uncountable pairwise compatible subset.

We now give two applications.

Theorem 1. Assume Martin's axiom for Borel ccc posets and let $T$ be a tree of size $<\mathfrak{c}$. If there is strictly increasing $f: T \rightarrow \mathbb{R}$, then there is such an $f$ with range included in $\mathbb{Q}$.

Proof. Every such tree is embeddable into the set of all well-ordered sets of rationals as a subposet of $\pi \mathbb{Q}$. Now apply MA for $\mathscr{P}_{0}^{<\omega}$.

Theorem 2. If every Borel ccc poset has property $K$, then every subset of $\omega^{\omega}$ of size $\aleph_{1}$ is bounded in the ordering of eventual dominance.

Proof. This follows from Claim 5. 
Note that $\mathscr{P}_{1}$ is the set of all finite homogeneous sets for certain partition on $\mathscr{S}$. Unfortunately, the partition in question is not open which would give the construction an added interest (see $[4, \S 8])$. But we shall see now that if we restrict the partition to a certain subset $\mathscr{S}^{*}$ of $\mathscr{S}$, it becomes open. The set $\mathscr{S}^{*}$ will be rich enough to provide the associated poset $\mathscr{P}_{1}^{*}$ with all the properties of $\mathscr{P}_{1}$. To define $\mathscr{S}^{*}$, we shall associate in a uniform way to every real $f$ in $\omega^{\omega}$ a sequence $\left\{f_{i}\right\}$ converging to $f$ as follows. Let $n_{0}<n_{1}<\cdots$ be the list of all $n$ such that $f(2 n+1) \neq 0$. For a given $i$, the real $f_{i}$ is determined by letting $f_{i} \uparrow n_{k}=f \uparrow n_{k}$ and

$$
f_{i}\left(n_{k}+j\right)=f\left(2^{i+1}\left(2 n_{k}+2 j+1\right)\right) \text {, }
$$

where $k=k(i)$ is minimal with the property

$$
f\left(2 n_{0}+1\right)+\cdots+f\left(2 n_{k}+1\right)>i
$$

if such a $k$ does not exist, let $f_{i}=f$. The partition $\left[\omega^{\omega}\right]^{2}=K_{o} \cup K_{c}$ is defined by $\{f, g\} \in K_{o}$ iff $f \neq g_{i}$ and $g \neq f_{j}$ for all $i$ and $j$. Note that this is an open partition in the sense that $K_{o}$ is an open subset of the plane and that, moreover, this is a ccc partition since, ignoring the $f$ 's for which $k(i)$ is not always defined, the set of all finite $o$-homogeneous sets is a subposet of $\mathscr{P}_{1}$. The argument from Claim 4 shows that we cannot cover the reals by countably many $o$-homogeneous sets. To get the analogue of Claim 5, working as in [4, 2.1], we fix an increasing and unbounded $\omega_{1}$-sequence $\left\{a_{\alpha}\right\}$ in $\omega^{\omega}$. Now recursively on $\beta$, construct $f_{\beta}$ in $\omega^{\omega}$ such that $a_{\beta}=\left(f_{\beta}\right)_{0}$ and such that if $\alpha<\beta$, and if $f_{\beta}\left(\triangle\left(f_{\alpha}, f_{\beta}\right)\right) \geq e(\alpha, \beta)$, then there is an $i$ such that $f_{\alpha}=\left(f_{\beta}\right)_{i}$ $(\triangle(f, g)$ is the minimal $n$ such that $f(n) \neq g(n))$. Note that $\left\{f_{\alpha}: \alpha \in A\right\}$ is unbounded in $\omega^{\omega}$ for every uncountable $A \subseteq \omega_{1}$, so the argument of [4, 2.1] shows that in any such $A$ we can find $\alpha<\beta$ such that $f_{\alpha}=\left(f_{\beta}\right)_{i}$ for some $i$, i.e., that $\left\{f_{\alpha}, f_{\beta}\right\} \in K_{c}$. This gives an uncountable set of reals with no uncountable homogeneous subset. Thus we have proved the following

Theorem 3. There is an open ccc partition $[\mathbb{R}]^{2}=K_{o} \cup K_{c}$ such that the reals cannot be covered by countably many o-homogeneous sets and such that if $\mathfrak{b}=\omega_{1}$ some uncountable set of reals contains no uncountable homogeneous subset.

In general, for partitions of the form $[X]^{2}=K_{0} \cup K_{1}$ being ccc is a considerable strengthening of the fact that there is no uncountable 1-homogeneous set. The following simple fact shows that in the situation of Theorem 3 these two conditions are equivalent.

Proposition 1. An open partition of the form $[\mathbb{R}]^{2}=J_{o} \cup J_{c}$ is ccc iff there is no uncountable $H \subseteq \mathbb{R}$ such that $[H]^{2} \subseteq J_{c}$. 


\section{REFERENCES}

1. M. Bell, Two Boolean algebras with extreme cellular and compactness properties, Canad. J. Math. 35 (1983), 824-838.

2. W. W. Comfort and S. Negrepontis, Chain conditions in topology, Cambridge Univ. Press, 1982.

3. S. Todorcevic, Remarks on cellularity in products, Compositio Math. 57 (1986), 357-372.

4. __ Partition problems in topology, Amer. Math. Soc., Providence, RI, 1988.

5. $\ldots$ Trees and linearly ordered sets, Handbook of Set-Theoretic Topology (K. Kunen and J. E. Vaughan, eds.), Elsevier Science Publishers, 1984.

Mathematical Sciences Research Institute, 1000 Centennial Drive, Berkeley, CaliFORNIA 94720

Current address: Mathematical Institute, Belgrade, Yugoslavia 